\title{
NOTE
}

\section{Preliminary organic compound analysis of microparticles returned from Asteroid 25143 Itokawa by the Hayabusa mission}

\author{
H. Naraoka, ${ }^{1 *}$ H. Mita, ${ }^{2}$ K. Hamase,${ }^{3}$ M. Mita,${ }^{4}$ H. Yabuta,${ }^{5}$ K. Saito,${ }^{6}$ K. Fukushima,${ }^{6}$ F. Kitajima, ${ }^{1}$ \\ S. A. SANDFOrd,${ }^{7}$ T. NAKAMURA,${ }^{8}$ T. NOGUChI,${ }^{9}$ R. OKAZAKI, ${ }^{1}$ K. NAGAO,${ }^{10}$ M. EBIHARA, ${ }^{11}$ H. Yurimoto,,${ }^{12}$

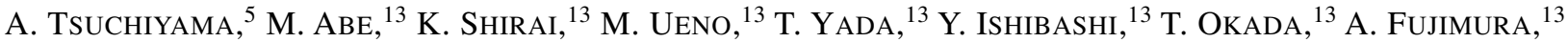 \\ T. MUKaI, ${ }^{13}$ M. YOSHIKAWA ${ }^{13}$ and J. KAWAGUCHI ${ }^{13}$ \\ ${ }^{1}$ Department of Earth and Planetary Sciences, Kyushu University, Fukuoka 812-8581, Japan \\ ${ }^{2}$ Department of Life, Environment and Materials Science, Fukuoka Institute of Technology, Fukuoka 811-0295, Japan \\ ${ }^{3}$ Graduate School of Pharmaceutical Sciences, Kyushu University, Fukuoka 812-8582, Japan \\ ${ }^{4}$ Frontier Science Div., Shiseido Co. Ltd., Minato-ku, Tokyo 105-0021, Japan \\ ${ }^{5}$ Department of Earth and Space Sciences, Osaka University, Toyonaka, Osaka 560-0043, Japan \\ ${ }^{6}$ Graduate School of Bioagricultural Sciences, Nagoya University, Nagoya, Aichi 464-8601, Japan \\ ${ }^{7}$ Astrophysics Branch, NASA-Ames Research Center, Moffett Field, CA 94035, U.S.A. \\ ${ }^{8}$ Department of Earth and Planetary Material Sciences, Tohoku University, Sendai, Miyagi 980-8578, Japan \\ ${ }^{9}$ College of Science, Ibaraki University, Mito, Ibaraki 310-8512, Japan \\ ${ }^{10}$ Geochemical Research Center, The University of Tokyo, Bunkyo-ku, Tokyo 113-0033, Japan \\ ${ }^{11}$ Department of Chemistry, Tokyo Metropolitan University, Hachioji, Tokyo 192-0397, Japan \\ ${ }^{12}$ Department of Earth and Planetary Sciences, Hokkaido University, Sapporo 060-0810, Japan \\ ${ }^{13}$ Institute of Space and Astronautical Science, Japan Aerospace Exploration Agency (ISAS/JAXA),
} Sagamihara, Kanagawa 229-8510, Japan

(Received May 10, 2011; Accepted September 7, 2011)

\begin{abstract}
Microparticles recovered from the Asteroid 25143 Itokawa surface by the Hayabusa mission have been examined for the occurrence of soluble organic compounds. After five individual particles ( 50 to $100 \mu \mathrm{m}$ in diameter) were rinsed with organic solvents on a diamond plate, two extracts were hydrolyzed with hydrochloric acid for amino acid analysis (AAA), and three extracts were combined for time of flight-secondary ion mass spectrometry (ToF-SIMS) to look for other organic compounds, including polycyclic aromatic hydrocarbons. The organic compounds detected by both methods have the same concentrations as those in blank levels, indicating that indigenous organic compounds are not found in this study. Based on the sensitivities of AAA and ToF-SIMS with the reference sample analyses, the concentrations of indigenous organics in the samples are below part-per-million (ppm), if present.
\end{abstract}

Keywords: Hayabusa spacecraft, Itokawa, microparticles, amino acid analysis, ToF-SIMS analysis

\section{INTRODUCTION}

Many kinds of organic compounds are observed in the interstellar medium as well as in extraterrestrial Solar System materials. In particular, various organic compounds including amino acids are reported from carbonaceous chondrites, which may have connections to emergence of life on the primitive Earth (e.g., Pizzarello, 2004; Martins, 2011). So far, materials collected by sample-return missions have not been exam-

*Corresponding author (e-mail: naraoka@geo.kyushu-u.ac.jp)

Copyright $@ 2012$ by The Geochemical Society of Japan. ined by organic analysis except for lunar soils collected by the Apollo Program (Harada et al., 1971) and cometary materials collected by the Stardust mission (Sandford $e t$ $a l .$, 2006). The Hayabusa mission has provided the first opportunity to examine the occurrence of organic matter on the surface of an asteroid without terrestrial contaminants. Asteroid 25143 Itokawa is an S-type asteroid, which is likely to be a parent body of LL5-6 ordinary chondrites (Abe et al., 2006; Okada et al., 2006). The collected particles consist mainly of olivines and pyroxenes having the same chemical and isotopic characteristics from those of LL4-6 chondrites (Nakamura et al., 2011; Yurimoto et al., 2011). Because ordinary chondrites of petrographic grade 4-6 have generally experienced high metamorphic 


\section{RA-QD02-00XX}
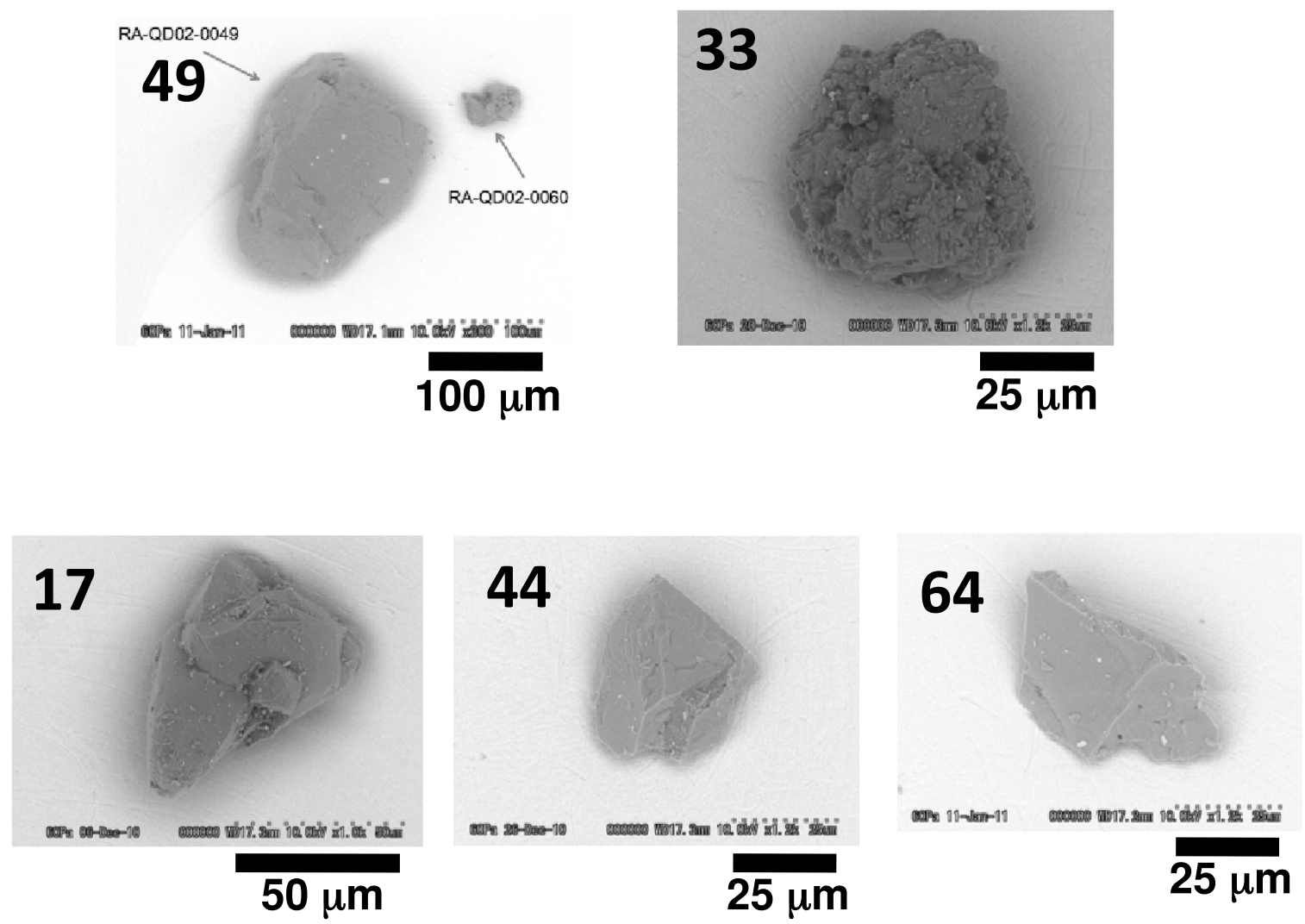

Fig. 1. SEM images of microparticles used in this study. The particle number XX denotes RA-QD02-00XX.

temperature ( $\sim 600$ to $950^{\circ} \mathrm{C}$; Huss et al., 2006), and because the equilibrated particles are likely to be heated up to $\sim 800^{\circ} \mathrm{C}$ by mineral assemblage (Nakamura et al., 2011), the Itokawa's rock would be originally depleted in volatile organic compounds. Even though $\sim$ ppb level of amino acids was reported in Antarctic LL5 chondrites (Botta et al., 2008), the detected amino acids may be contaminants from Antarctic ice. Or carbonaceous particles with amino acids may be incorporated into the meteorite parent body after thermal metamorphism.

The surface of Itokawa may not be a good place to find organic matter, since the surface layer of the asteroid is subjected to radiation exposure (i.e., space weathering), which would result in decomposition of organic matter. At the same time, however, not only carbonaceous particles including cometary dusts may be transported from space, but also the organic precursors such as $\mathrm{CN}$ could be implanted into the surface particles by solar winds, which may result in amino acids by hydrolysis. Therefore, the existence of organic compounds is possi- ble at the surface of Itokawa. Actually, indigenous amino acids are found even in lunar surface soils (Harada et al., 1971; Brinton and Bada, 1996). Harada et al. (1971) first identified various amino acids such as glycine (Gly), alanine (Ala), and aspartic acid (Asp) at $\sim$ ppb level by ninhydrin detection using an ion-exchange amino acid analyzer. The result was confirmed using reverse phase high-performance liquid chromatography with fluorescence detection (Brinton and Bada, 1996). Cometary glycine was also identified in acid hydrolyzed hot water extracts of collector aerogel and foil returned by the Stardust mission (Elsila et al., 2009). Antarctic carbonaceous micrometeorites generally contain Gly as the most abundant amino acid followed by Ala with a few samples enriched in $\alpha$-aminoisobutyric acid (AIB) up to $\sim 280 \mathrm{ppm}$ (Brinton et al., 1998), which is not usually observed in terrestrial amino acids. Such a high AIB concentration is 10-100 times the level of that found in CM chondrites (Shimoyama et al., 1979; Ehrenfreund et al., 2001). In addition, polycyclic aromatic hydrocarbons (PAHs) are 


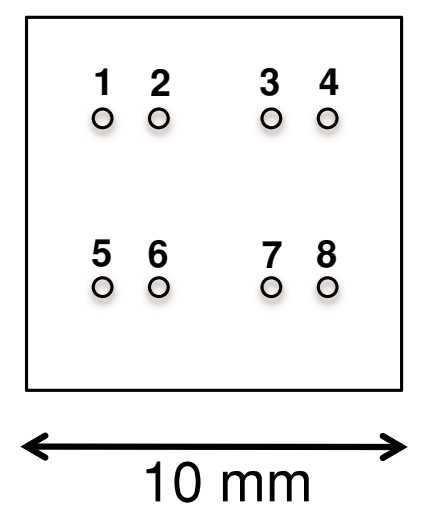

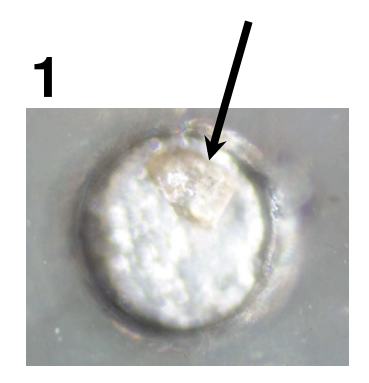

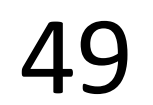

5

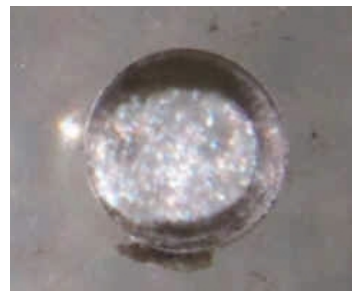

Blank
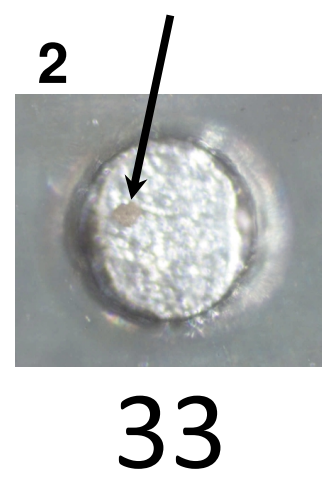

6

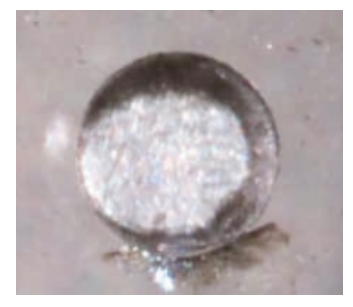

Blank

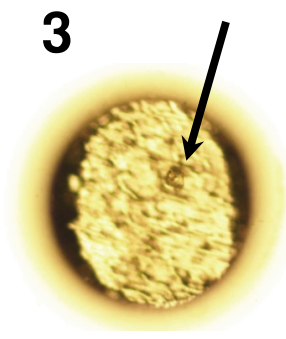

17

7

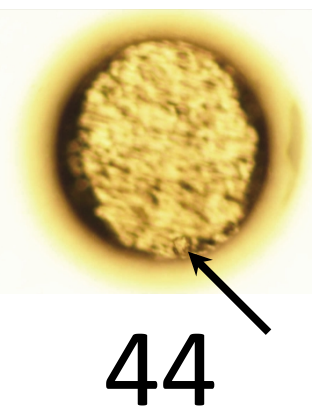

4

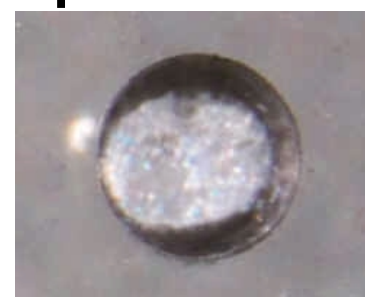

Blank

8

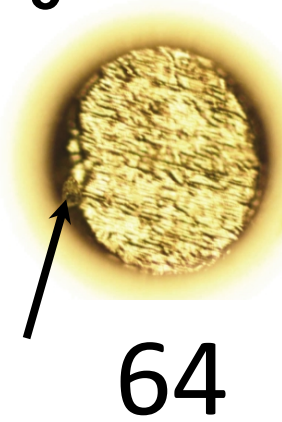

Fig. 2. Schematic view of a diamond plate containing 8 holes $(500 \mu \mathrm{m}$ in diameter and $200 \mu \mathrm{m}$ in depth each) and photos of each hole used for solvent extraction. Blank holes were used for procedural blanks.

reported in carbonaceous chondrites (Naraoka et al., 2000) and IDPs (Clemett et al., 1993).

The compound distributions may clarify origins of organic compounds at the surface of Itokawa. If Gly is abundant, as observed in lunar soils (Harada et al., 1971; Brinton and Bada, 1996) and cometary dusts (Sandford et al., 2006), hydrogen cyanide (HCN) may contribute to the amino acid precursors. In the case of anhydrous minerals, the HCN may be implanted by solar wind (e.g., Fox et al., 1976). If $\alpha$-aminoisobutyric acid (AIB) is abundant as observed in some CM chondrites, the meteoritic source could be delivered from aqueously altered parent bodies after a Strecker-type reaction with ketones and $\mathrm{HCN}$ followed by hydrolysis. In such a case, the organic compounds survived upon impact. In this study, we report on the first analyses of complex organic compounds in microparticles from asteroid Itokawa returned by the Hayabusa mission.

\section{SAMPleS AND Analytical MethodS}

\section{Samples}

Five particles (RA-QD02-0017, -0033, -0044, -0049, and -0064) were allocated for organic and carbonaceous 
material analyses (Fig. 1). The -0017 particle is triangle in shape with $\sim 50 \mu \mathrm{m}$ in a long dimension and has $\mathrm{Mg}$, $\mathrm{Si}$, and $\mathrm{O}$ peaks in scanning electron microscopy with energy dispersive X-ray spectrometry (SEM-EDS), implying it is a $\mathrm{Mg}$-silicate. The -0033 particle is roundshape ( $\sim 50 \mu \mathrm{m}$ in diameter) and porous. The SEM-EDS analysis implies that it is predominantly an $\mathrm{Fe}, \mathrm{Mg}$ silicate with a tiny amount of carbon. The -0044 particle is the smallest $(\sim 30 \mu \mathrm{m})$ one in this study, consisting mainly of $\mathrm{Fe}, \mathrm{Mg}$-silicate with $\mathrm{Al}$ as measured by SEMEDS. The -0049 is the largest particle $(\sim 200 \times 100 \times 50$ $\mu \mathrm{m})$ in this study, similarly consisting of $\mathrm{Fe}, \mathrm{Mg}$-silicate with Al. The -0064 particle is $\sim 40 \mu \mathrm{m}$ in its long dimension, and is a $\mathrm{Mg}$ silicate similar to the -0017 particle. The semi-quantitative analysis using field emission-SEM/ EDS also indicates that major constituent minerals are Mg-rich olivine (-0017), Mg-rich olivine, and Mg-rich and low-Ca pyroxene with minor FeS and plagioclase (0033), Mg-rich olivine (-0044), Mg-rich olivine (-0049; Ebihara et al., 2011), and Mg-rich olivine (-0064). These samples were put on a diamond plate containing 8 holes (500 $\mu \mathrm{m}$ in diameter and $250 \mu \mathrm{m}$ in depth) by electrostatic manipulation on a clean bench in the curation facility at ISAS/JAXA. The samples were covered by a diamond plate for transportation to Kyushu University in a nitrogen gas-purged container (Fig. 2).

\section{Analytical methods}

The analytical scheme is shown in Fig. 3. After spectroscopic analyses including Raman and FT-IR spectroscopy (Kitajima et al., 2011), the particles were rinsed with small amount of dichloromethane (DCM)/ methanol $(\mathrm{MeOH})(1 / 1, \sim 0.2 \mu \mathrm{l} \times 3)$ on the plate in a clean bench. Ideally, even though it would be preferred to extract particles with hot water for amino acid analysis, it was prohibited to use water for the preliminary analysis because of probable aqueous weathering of original minerals used for other mineral studies after organic analysis. The extracts of -0033 and -0049 particles were hydrolyzed with $\sim 20 \mu$ of doubly distilled azeotrope- $\mathrm{HCl}$ $(c a .5 .8 \mathrm{M})$ in a glass ampoule at $105^{\circ} \mathrm{C}$ for $20 \mathrm{~h}$. The hydrolysate was dried in vacuo using a liquid-nitrogen trap followed by reaction with a fluorescence reagent (4fluoro-7-nitro-2,1,3-benzoxadiazole, Hamase et al., 2010) to assist with amino acid analysis with enantiomeric separation. A highly sensitive detection limit ( $\sim$ sub femto mole) was achieved by two-dimensional high performance liquid chromatography with fluorescence detection (2D-HPLC/FD; NANOSPACE SI-II series, Shiseido, Hamase et al., 2010). A microbore-monolithic ODS column was used as the first column for each amino acid separation followed by chiral-stationary phase-bearing narrowbore-Sumichiral OA-2500R as the second column for its enantiomeric separation. In this study, the abun-

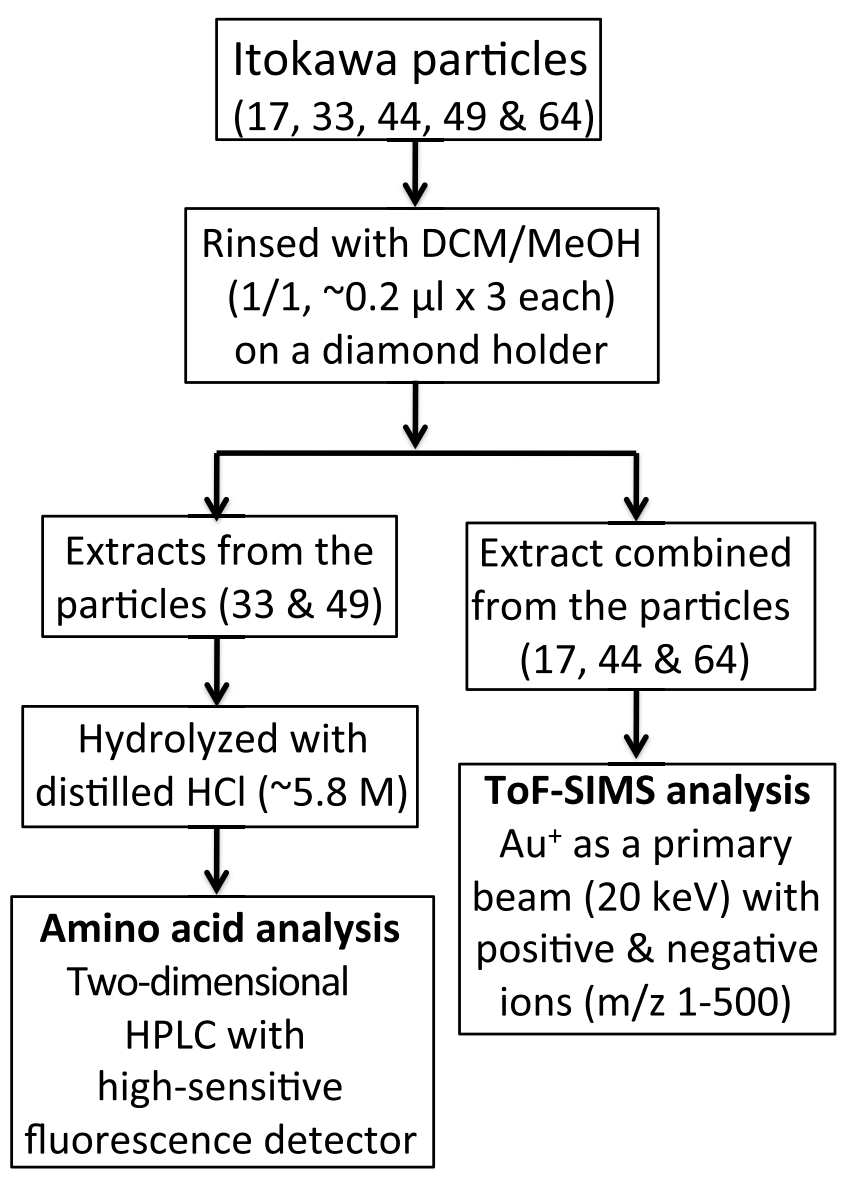

Fig. 3. Analytical scheme.

dance of Gly, L,D-Ala, AIB, and L,D-isovaline (Isoval) is examined, because Gly and Ala are abundant amino acids in most extraterrestrial materials, and AIB and Isoval are free of terrestrial materials but having characteristic signatures in carbonaceous meteorites. AIB is often most abundant amino acid in some CM2 chondrites (e.g., Shimoyama et al., 1985), and L-Isoval is enriched relative to D-Isoval for aqueously altered meteorites (Pizzarello et al., 2003; Glavin and Dworkin, 2009).

The extracts of $-0017,-0044$, and -0064 particles were combined into a hole $(c a .500 \mu \mathrm{m}$ in diameter and $c a$. $200 \mu \mathrm{m}$ in depth) on a quartz plate to analyze for other organic compounds including a search for PAHs using time of flight-secondary ion mass spectrometry (ToFSIMS) at Nagoya University. The ToF-SIMS analysis was performed using by $\mathrm{Au}^{+}$as a primary ion at $22 \mathrm{eV}$ and using an ULVAC Phi III to collect ions from $\mathrm{m} / \mathrm{z}=2$ to 500. A $300 \mu \mathrm{m} \times 300 \mu \mathrm{m}$ area was rastered for about 10 15 minutes until the total ion current was 5-6 millions as measured using a dual micro channel plate.

Three vacant holes of the diamond plate were used for procedural blanks. All glassware and the quartz plate 


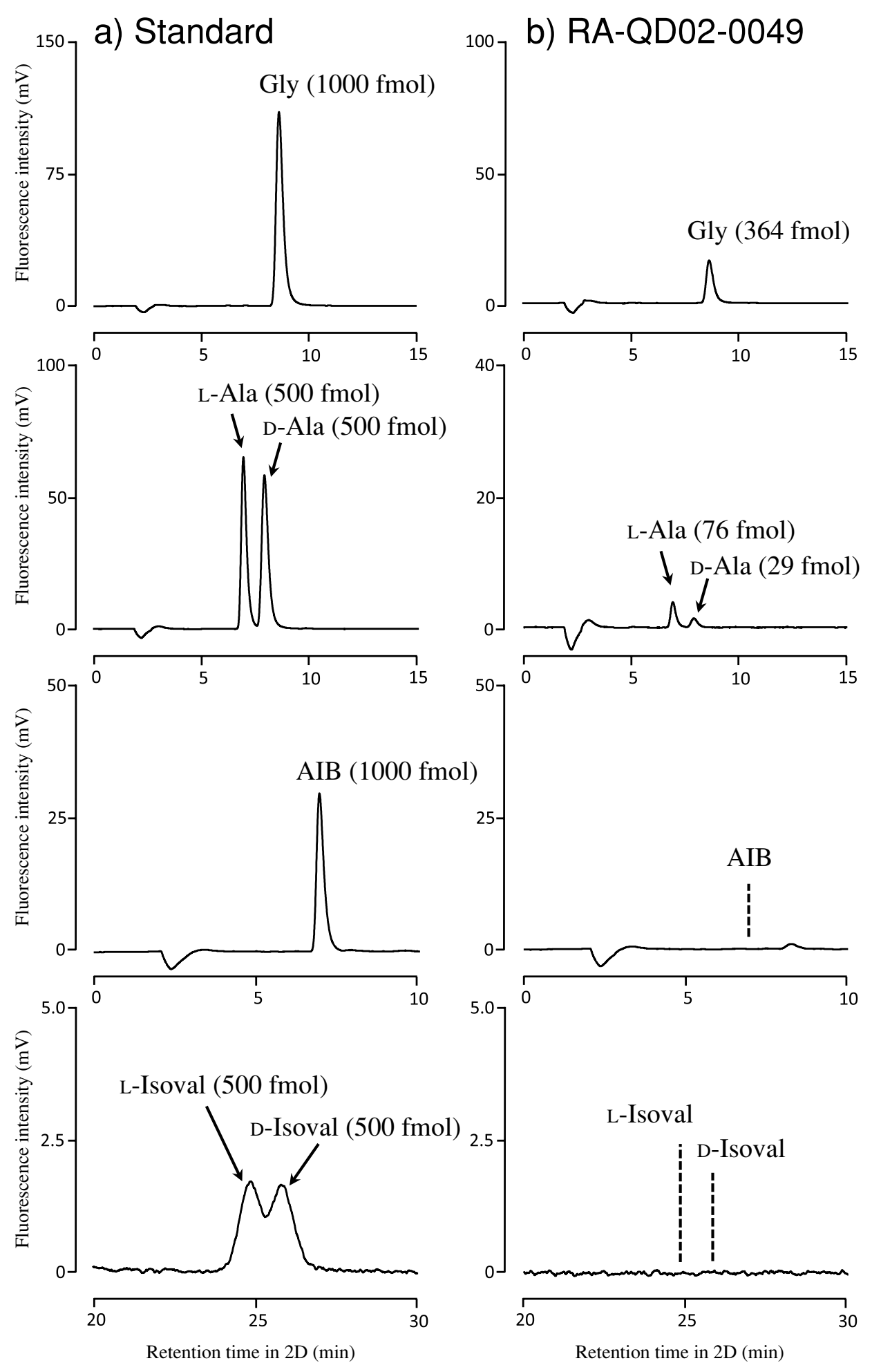

Fig. 4. Amino acid chromatograms of glycine (Gly), alanine (L,D-Ala), $\alpha$-aminoisobutyric acid (AIB) and isovaline (L,D-Isoval). a) standard, b) RA-QD02-0049, c) RA-QD02-0033, d) blank, and e) Y-791191. Samples of RA-QD02-0049, RA-QD02-0033, blank and $Y-791191$ were injected by $40 \mu \mathrm{l}$ of whole solution $(100 \mu \mathrm{l})$. 


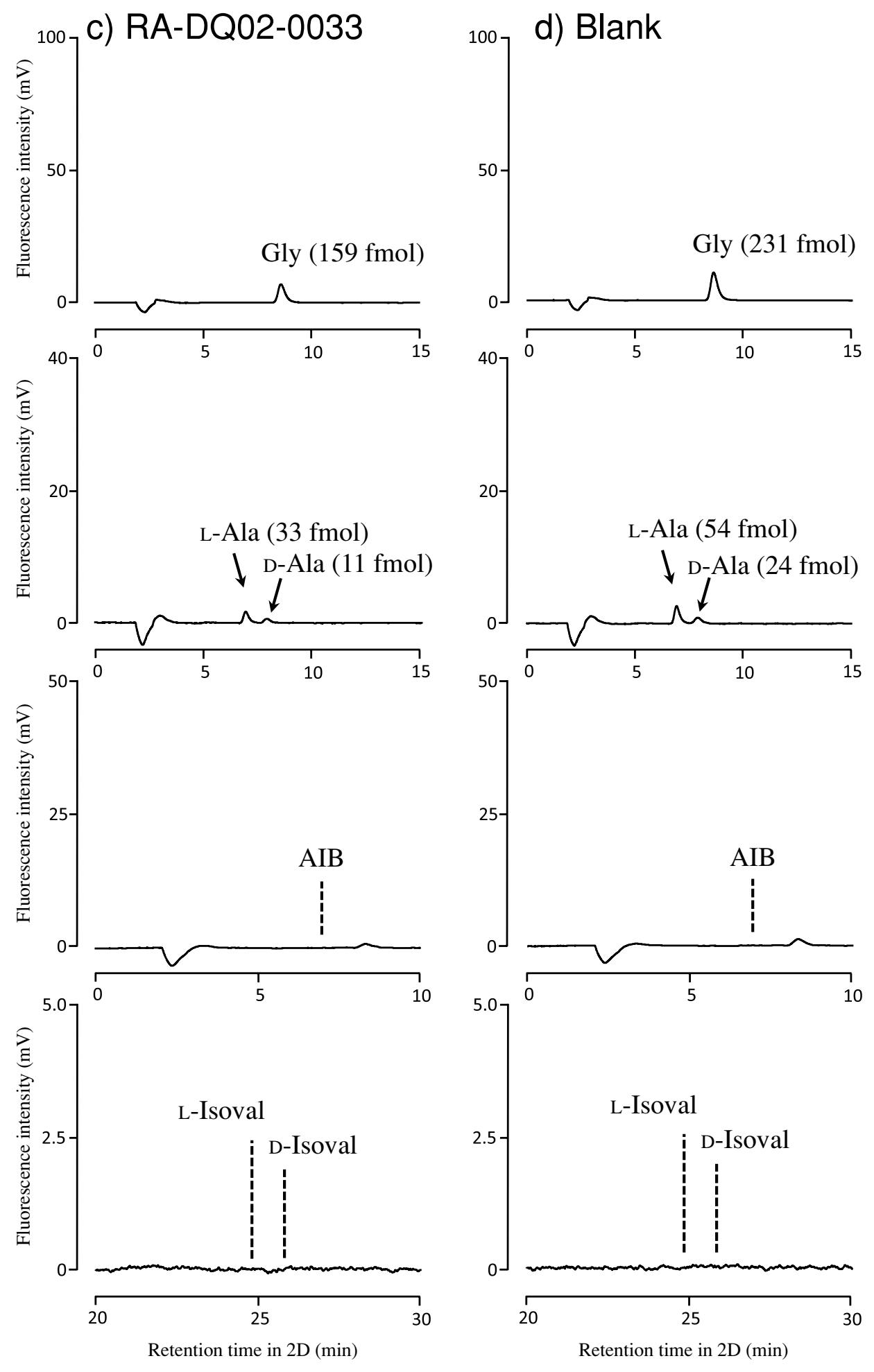

Fig. 4. (continued). 


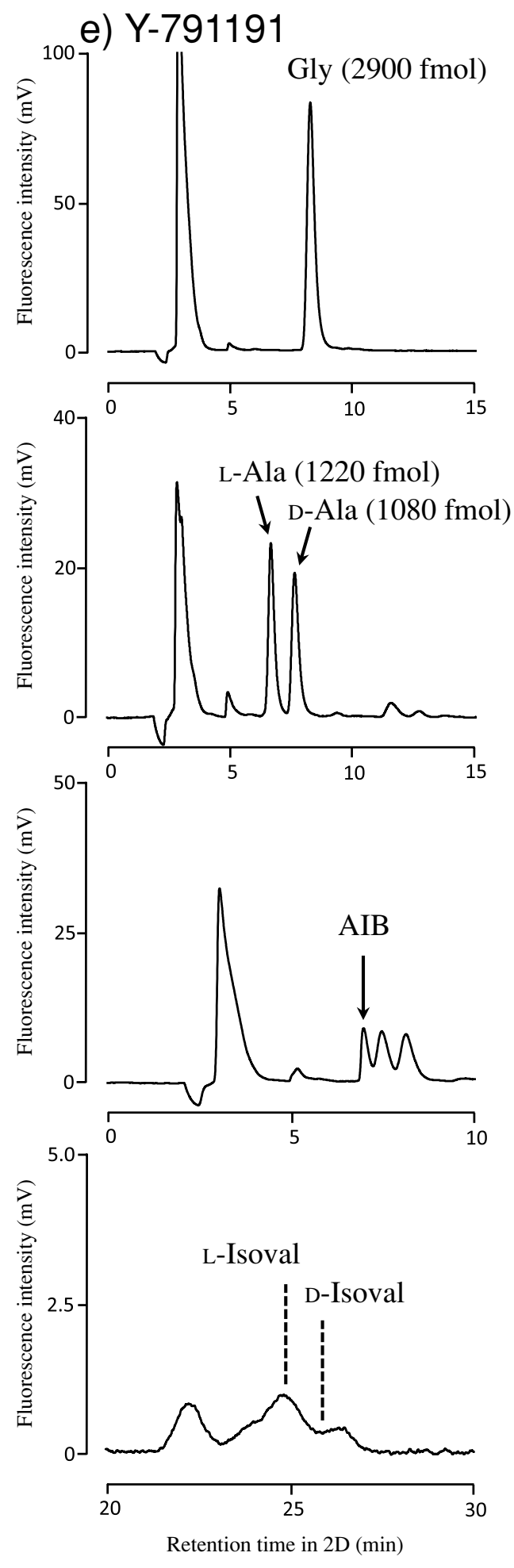

Fig. 4. (continued). were cleaned and rinsed with deionized water (18.2 M $\Omega$ ) followed by wrapped with aluminum foil and baked in an air oven at $450^{\circ} \mathrm{C}$ for $3 \mathrm{~h}$ prior to use to remove organic contaminants. The matrix grains $(\sim 50 \mu \mathrm{m})$ of the Yamato(Y)791191 carbonaceous chondrite (CM2) was also analyzed to pursue the detection limit, using the direct $\mathrm{HCl}$ hydrolysis of grains for amino acid analysis and the DCM/MeOH extraction for ToF-SIMS analysis.

\section{RESULTS}

\section{Amino acid analysis}

Figure 4 shows amino acid chromatograms of the -0033 and -0049 extracts with their procedural blanks as well as comparison with amino acid standards and the extract of Y791191. While Gly and Ala were identified in all samples, AIB and Isoval were detected only from Y791191. Based on the peak area relative to the standard solution, the Gly content of the - 0049 extract is $364 \mathrm{fmol}$, which is slightly higher than that of procedural blank (231 fmol). However, the Gly content of -0033 extract (159 fmol) is less than that of procedural blank. Similarly, while the L- and D-Ala contents of -0049 extract (76 and 29 fmol, respectively) are slightly higher than those of procedural blank (54 and 22 fmol, respectively), the L- and D-Ala contents of -0033 extract (33 and 11 fmol, respectively) are less than those of procedural blank. L-Ala is always enriched relative to D-Ala. On the other hand, as shown in Fig. 4e, 100 ppm level of Gly, L,D-Ala and AIB can be clearly quantified in the $\mathrm{HCl}$-extract sample of three $\sim 50 \mu \mathrm{m}$ grains of Y791191, where the Ala is present as a nearly racemic mixture.

\section{ToF-SIMS analysis}

Mass spectra of ToF-SIMS are shown in Figs. 5 and 6 . The predominant peaks of positive ions are at $\mathrm{m} / \mathrm{z}=27$ and 28 (Fig. 5), which are attributable to the aluminum from foil used for baking and silicon from the quartz plate, respectively. The negative ions show a strong peak of $\mathrm{m} /$ $z=16$ derived from oxygen from quartz plate (Fig. 6). Even though $\mathrm{Mg}$ is a main constituent of particles identified by SEM-EDS, the Mg peak $(m / z=24)$ is not observed in this extract. In addition, as the Mg mapping $(\mathrm{m} /$ $z=24$ ) did not show any Mg-enriched region, the extract did not contain any debris from the particle. In contrast to the strong mass peaks ( 2 to 3 million counts) of $\mathrm{Al}, \mathrm{Si}$, and $\mathrm{O}$, many small peaks ( $<6000$ counts) are observed between $\mathrm{m} / \mathrm{z}$ values of 100 to 500 for both ions, which are probably assigned to organic compounds.

\section{DISCUSSION}

Amino acid distribution

The presence of Gly and DL-Ala in the particles is 


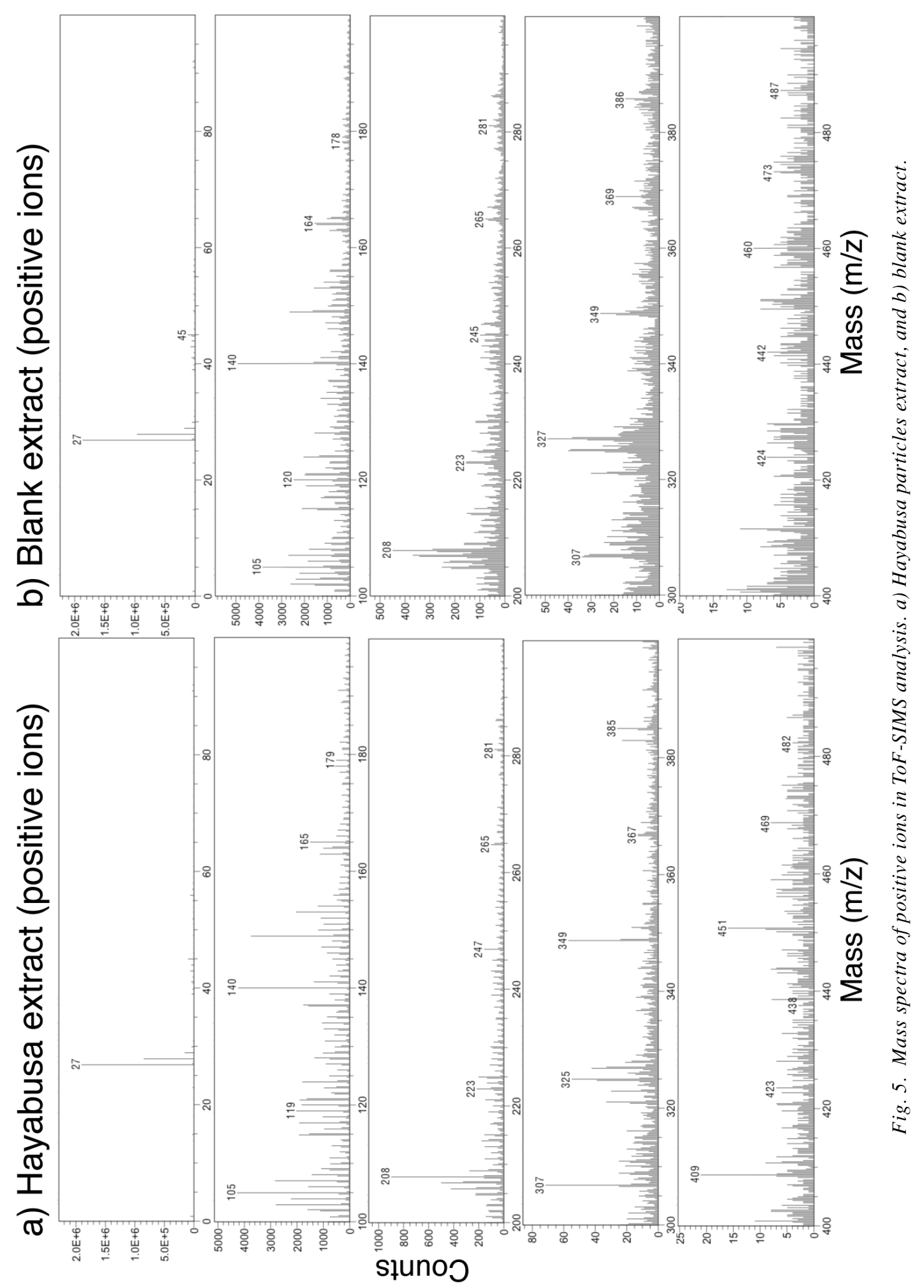




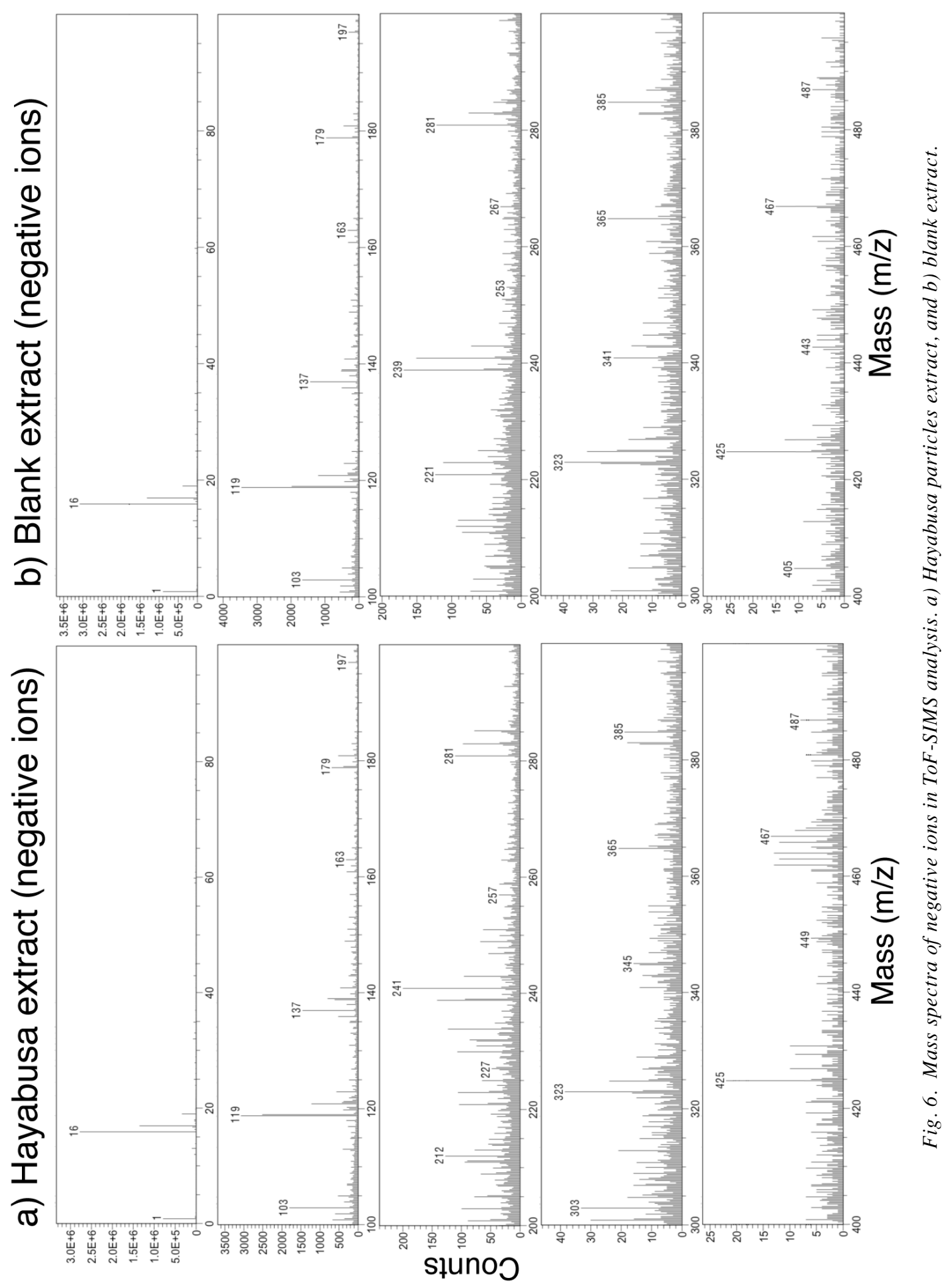


consistent with the amino acid distribution of hydrolysates of lunar soils (Harada et al., 1971; Brinton and Bada, 1996). However, Gly and L-Ala are the most common amino acids in terrestrial environments and they are detected in similar amounts for the procedural blanks. Even though D-amino acids, including D-Ala, are often regarded as a sign for extraterrestrial amino acids (e.g., Pizzarello, 2004), D-Ala is observed in the particles at similar amounts relative to blank levels, implying that D-Ala is not an indicator of extraterrestrial origin in this study. Assuming the density of particles as $2.5 \mathrm{~g} / \mathrm{cm}^{3}$, the -0033 and -0049 particles are estimated to be $\sim 0.16 \mu \mathrm{g}$ and $\sim 1.0 \mu \mathrm{g}$, respectively. If the Gly content is literally divided by the particle weight, the concentrations range from $\sim 150 \mathrm{fmol} / \mu \mathrm{g}(-0049)$ to $\sim 990 \mathrm{fmol} / \mu \mathrm{g}(-0049)$, indicating $\sim 11$ to $\sim 73 \mathrm{ppm}$ for these two particles, respectively. The Gly of $\sim$ ppm level is anomalously high for the particles consisting of $\mathrm{Mg}$-silicate, as lunar soils contain Gly only in ppb level (Harada et al., 1971; Brinton and Bada, 1996). Therefore, Gly and DL-Ala detected in this study are mostly contaminants. The lack of detection of AIB, a non-proteinaeous and abundant amino acid in CM chondrites, may also support this consideration.

In the $\mathrm{HCl}$-extract sample of three $\sim 50 \mu \mathrm{m}$ grains of Y791191 chondrite (CM2), 100 ppm level of Gly, L,DAla, and AIB can be clearly quantified using the present 2D-HPLC system as shown in Fig. 4e. About 100 ppm level of amino acids may be too high for CM chondrites. If the three $\sim 50 \mu \mathrm{m}$ size particles used weigh totally 1 $\mu \mathrm{g}$, the content of glycine is $216 \mathrm{ppm}$, which is 20 times higher than that of glycine in Y791198, which yielded the most abundant amino acids among CM chondrites (Shimoyama et al., 1985). So far, the meteoritic amino acids have been analyzed using bulk meteorite powders $(\sim \mathrm{mg}$ to $\sim \mathrm{g}$ scale $)$. However, the amino acid and its precursors are distributed heterogeneously in the meteorite, where the phyllosilicate part (known as black matrix) seems to contain more amino acids than chondrules and CAIs. As we extracted the particle sample of black matrix directly with hydrochloric acid, the observed content of amino acids may not be extraordinarily large. In a future study, we will analyze particle samples separated from meteorites as references using various extraction methods. In addition, the observed Ala is present as a nearly racemic mixture, far from terrestrial contamination. This suggests that, if a $\sim 100 \mu \mathrm{m}$ of carbonaceous grain is analyzed, it is likely to detect indigenous amino acids.

\section{Compound distribution by ToF-SIMS}

The mass spectra of ToF-SIMS are fundamentally the same between the extract and procedural blank, and both mainly show peaks derived from the quartz plates (pre- dominant $\mathrm{m} / \mathrm{z}=28$ with minor $\mathrm{m} / \mathrm{z}=43$ and 45 in positive ions and minor $m / z=25,28,32,35$ and 60 in negative ions from methytrimethoxysilane) and aluminum foils (predominant $\mathrm{m} / \mathrm{z}=27$ in positive ion with minor $\mathrm{m} / \mathrm{z}=$ 43 and 59 in negative ions from $\mathrm{Al}_{2} \mathrm{O}_{3}$ ). In addition, small peaks are assigned to phthalates (mainly as dimethylphthalate), which have $m / z=55,57,73$ and 149 in positive ions and $\mathrm{m} / \mathrm{z}=60,61$ and 77 in negative ions. As phthalates are commonly used as plasticizers, they are probably contaminants introduced to the samples during procedures in the laboratory. Many small peaks of $\mathrm{m} / \mathrm{z}>$ 100 are not currently assigned to specific organic compounds. However, as substantial differences are not observed between the particle extract and procedural blank, indigenous compounds have not been found from the Hayabusa particles.

\section{Future study}

The presence of organic compounds is possible on the surface of Itokawa, even though its evidence is not obtained in this study. The detection is highly dependent on the concentration of compounds in the particles as well as the sample amount available for the analysis. High sensitivity and low procedural blank are also critical.

In the case of the lunar soil study, $1 \mathrm{~g}$ of sample was used to detect for $\sim$ ppb amino acids (Harada et al., 1971; Brinton and Bada, 1996). A 1 g of lunar samples may contain small numbers of carbonaceous particles, which could yield the proper amount of organic compounds detected. Although a high absolute sensitivity $(\sim 0.1 \mathrm{fmol})$ is achieved by 2D-HPLC/FD in this study, the small amounts of sample available for this study (1 olivine grain with $<1 \mu \mathrm{g}$ ) result in a comparable detection limit of $\sim$ ppb level. To overcome this difficulty, it will be necessary to reduce the background level during analytical procedures, including contamination from the atmosphere as well as from solvents and chemical reagents. In particular, as the analytical procedure does not induce racemization of amino acids, the detected D-Ala of this study is derived from chemical reagents and/or a memory of amino acid analyzer. Such an analytical background must be improved. It is also important to evaluate possible organic contaminants from the spacecraft and/or sample acquisition processes after the establishment of procedural blank.

The extraction method will also need to be improved, as hot water extraction with acid hydrolysis gives effective yields of amino acids. As the direct $\mathrm{HCl}$ extraction of carbonaceous grains gave larger amount of amino acids, such a method may be further effective. For ToFSIMS analysis, instead of solvent extraction in this study, the particle will be directly studied for both soluble and insoluble organic compounds, which also can remove the issue of contamination added from solvents. 


\section{SUMMARY}

Amino acids such as glycine and alanine were detected only at procedural blank levels in two different grains from asteroid Itokawa using 2D-HPLC with highsensitive fluorescence detection. ToF-SIMS analysis also gave no substantial difference between sample extracts and blanks. Indigenous organic compounds were therefore not identified in this study, and if present, the amino acid concentrations in these samples are at the $\sim$ ppm level or lower for the DCM/MeOH extract. The particle amount available for this study was very limited. Further analyses of acid hydrolyzed hot water extracts of Itokawa samples using larger sample sizes and the least minimum procedural blank may be required in order to detect trace levels of indigenous amino acids. A direct particle analysis by ToF-SIMS without solvent extraction is also preferred. In addition, carbonaceous microparticles will be searched in the Itokawa samples for organic compound analyses.

Acknowledgments - We are grateful to Profs. D. P. Glavin, S. Pizzarello and G. D. Cody for helpful comments to revise the manuscript. This study was partially supported by Grant-in-Aid for Scientific Research (B) no 22340166 from Japan Society for the Promotion of Science and Research Fund form Consortium Fukuoka to HM and KH.

\section{REFERENCES}

Abe, M., Takagi, Y., Kitazato, K., Abe, S., Hiroi, T., Vilas, F., Clark, B. E., Abell, P. A., Lederer, S. M., Jarvis, K. S., Mimura, T., Ueda, Y. and Fujiwara, A. (2006) Near-infrared spectral results of asteroid Itokawa from the Hayabusa spacecraft. Science 312, 1334-1338.

Botta, O., Martins, Z., Emmeneggers, C., Dworkin, J. P., Glavin, D. P., Harvey, R. P., Zenobi, R., Bada, J. L. and Ehrenfreund, P. (2008) Polycyclic aromatic hydrocarbons and amino acids in meteorites and ice samples from LaPaz Icefield, Antarctica. Meteor. Planet. Sci. 43, 1465-1480.

Brinton, K. L. F. and Bada, J. L. (1996) A reexamination of amino acids in lunar soils: Implications for the survival of exogenous organic material during impact delivery. Geochim. Cosmochim. Acta 60, 349-354.

Brinton, K. L. F., Engrand, C., Glavin, D. P., Bada, J. L. and Maurette, M. (1998) A search for extraterrestrial amino acids in carbonaceous Antarctic micrometeorites. Orig. Life Evol. Biosphere 28, 413-424.

Clemett, S. J., Maechling, C. R., Zare, R. N., Swan, P. D. and Walker, R. M. (1993) Identification of complex aromatic molecules in individual interplanetary dust particles. Science 262, 721-725.

Ebihara, M., Sekimoto, S., Shirai, N., Hamajima, Y., Yamamoto, M., Kumagai, K., Oura, Y., Ireland, T. R., Kitajima, F., Nagao, K., Nakamura, T., Naraoka, H., Noguchi, T., Okazaki, R., Tsuchiyama, A., Uesugi, M., Yurimoto, H., Zolensky, M. E., Abe, M., Fujimura, A., Mukai, T. and Yada,
T. (2011) Neutron activation analysis of a particle returned from Asteroid Itokawa. Science 333, 1119-1121.

Ehrenfreund, P., Glavin, D. P., Botta, O., Cooper, G. and Bada, J. L. (2001) Extraterrestrial amino acids in Orgueil and Ivuna: Tracing the parent body of CI type carbonaceous chondrites. Proc. Natl. Acad. Sci. 98, 2138-2141.

Elsila, J. E., Glavin, D. P. and Dworkin, J. P. (2009) Cometary glycine detected in samples returned by Stardust. Meteor. Planet. Sci. 44, 1323-1330.

Fox, S. W., Harada, K. and Hare, P. E. (1976) Amino acid precursors in lunar fines: limits to the contribution of jet exhaust. aqueous extracts of Apollo 11 and Apollo 12 lunar fines. Geochim. Cosmochim. Acta 40, 1069-1071.

Glavin, D. P. and Dworkin, J. P. (2009) Enrichment of the amino acid L-isovaline by aqueous alteration on CI and CM meteorite parent bodies. Proc. Natl. Acad. Sci. 106, 5487-5492.

Hamase, K., Miyoshi, Y., Ueno, K., Han, H., Hirano, J., Morikawa, A., Mita, M., Kaneko, T., Lindner, W. and Zaitsu, K. (2010) Simultaneous determination of hydrophilic amino acid enantiomers in mammalian tissues and physiological fluids applying a fully automated micro-two-dimensional high-performance liquid chromatographic concept. $J$. Chromatogr. A 1217, 1056-1062.

Harada, K., Hare, P. E., Windsor, C. R. and Fox, S. W. (1971) Evidence for compounds hydrolysable to amino acids in aqueous extracts of Apollo 11 and Apollo 12 lunar fines. Science 173, 433-435.

Huss, G. R., Rubin, A. E. and Grossman, J. N. (2006) Thermal metamorphism in chondrites. Meteorites and the Early Solar System II (Lauretta, D. S. and McSween, H. Y., Jr., eds.), 567-586, University of Arizona Press, Tucson, AZ, U.S.A.

Kitajima, F., Kotsugi, M., Ohkochi, T., Naraoka, H., Ishibashi, Y., Abe, M., Fujimura, A., Okazaki, R., Yada, T., Nakamura, T., Noguchi, T., Nagao, K., Tsuchiyama, A., Mukai, T., Sandford, S. A., Okada, T., Shirai, K., Ueno, M., Yoshikawa, M. and Kawaguchi, J. (2011) A micro-spectroscopic approach to the carbonaceous matter in the particles recovered by the Hayabusa mission. Lunar Planet. Sci. 42, \#1855.

Martins, Z. (2011) Organic chemistry of carbonaceous chondrites. Elements 7, 35-40.

Nakamura, T., Noguchi, T., Tanaka, M., Zolensky, M. E., Kimura, M., Tsuchiyama, A., Nakato, A., Ogami, T., Ishida, H., Uesugi, M., Yada, T., Shirai, K., Fujimura, A., Okazaki, R., Sandford, S. A., Ishibashi, Y., Abe, M., Okada, T., Ueno, M., Mukai, T., Yoshikawa, M. and Kawaguchi, J. (2011) Itokawa dust particles: A direct link between S-Type asteroids and ordinary chondrites. Science 333, 1113-1116.

Naraoka, H., Shimoyama, A. and Harada, K. (2000) Isotopic evidence from an Antarctic carbonaceous chondrites for two reaction pathways of extraterrestrial PAH formation. Earth Planet. Sci. Lett. 184, 1-7.

Okada, T., Shirai, K., Yamamoto, Y., Arai, T., Ogawa, K., Hosono, K. and Kato, M. (2006) X-ray fluorescence spectrometry of asteroid Itokawa by Hayabusa. Science 312, 1338-1341.

Pizzarello, S. (2004) Chemical evolution and meteorites: An update. Orig. Life Evol. Biosphere 34, 25-34.

Pizzarello, S., Zolensky, M. and Turk, K. A. (2003) Nonracemic isovaline in the Murchison meteorite: Chiral distribution 
and mineral association. Geochim. Cosmochim. Acta 67, 1589-1595.

Sandford, S. A., Aléon, J., Alexander, C. M. O’D., Araki, T., Bajt, S., Baratta, G. A., Borg, J., Bradley, J. P., Brownlee, D. E., Brucato, J. R., Burchell, M. J., Busemann, H., Butterworth, A., Clemett, S. J., Cody, G., Colangeli, L., Cooper, G., D’Hendecourt, L., Djouadi, Z., Dworkin, J. P., Gianluca Ferrini, G., Fleckenstein, H., Flynn, G. J., Franchi, I. A., Fries, M., Gilles, M. K., Glavin, D. P., Gounelle, M., Grossemy, F., Jacobsen, C., Keller, L. P., Kilcoyne, D., Leitner, L., Matrajt, G., Meibom, A., Mennella, V., Mostefaoui, S., Nittler, L. R., Palumbo, M. E., Papanastassiou, D. A., Robert, F., Rotundi, A., Snead, C. J., Spencer, M. K., Stadermann, F. J., Steele, A., Stephan, T., Tsou, P., Tyliszczak, T., Westphal, A. J., Wirick, S., Wopenka, B., Yabuta, H., Zare, R. N. and Zolensky, M. E. (2006) Organics captured from comet $81 \mathrm{P} /$ Wild 2 by the
Stardust Spacecraft. Science 314, 1720-1724.

Shimoyama, A., Ponnamperuma, C. and Yanai, K. (1979) Amino acids in the Yamato carbonaceous chondrite from Antarctica. Nature 282, 1183-1186.

Shimoyama, A., Harada, K. and Yanai, K. (1985) Amino acids from the Yamato-791198 carbonaceous chondrite from Antarctica. Chem. Lett. 1985, 394-396.

Yurimoto, H., Abe, K., Abe, M., Ebihara, E., Fujimura, A., Hashiguchi, M., Hashizume, K., Ireland, T. R., Itoh, S., Katayama, J., Kato, C., Kawaguchi, J., Kawasaki, N., Kitajima, F., Kobayashi, S., Meike, T., Mukai, T., Nagao, K., Nakamura, T., Naraoka, H., Noguchi, T., Okazaki, R., Park, C., Sakamoto, N., Seto, Y., Takei, M., Tsuchiyama, A., Uesugi, M., Wakaki, S., Yada, T., Yamamoto, K., Yoshikawa, M. and Zolensky, M. E. (2011) Oxygen isotopic compositions of asteroidal materials returned from Itokawa by the Hayabusa mission. Science 333, 1116-1119. 